\title{
The Influence of Changes in Acute Training Load on Daily Sensitivity of Morning-Measured Fatigue Variables in Elite Soccer Players
}

\author{
Robin T. Thorpe, Anthony J. Strudwick, Martin Buchheit, Greg Atkinson, \\ Barry Drust, and Warren Gregson
}

\begin{abstract}
Purpose: To determine the sensitivity of a range of potential fatigue measures to daily training load accumulated over the previous 2, 3, and $4 \mathrm{~d}$ during a short in-season competitive period in elite senior soccer players $(\mathrm{N}=10)$. Methods: Total highspeed-running distance, perceived ratings of wellness (fatigue, muscle soreness, sleep quality), countermovement-jump height (CMJ), submaximal heart rate (HRex), postexercise heart-rate recovery (HRR), and heart-rate variability (HRV: Ln rMSSD) were analyzed during an in-season competitive period (17 d). General linear models were used to evaluate the influence of 2-, 3-, and 4-d total high-speed-running-distance accumulation on fatigue measures. Results: Fluctuations in perceived ratings of fatigue were correlated with fluctuations in total high-speed-running-distance accumulation covered on the previous $2 \mathrm{~d}(r=-.31$; small), $3 \mathrm{~d}$ ( $r=-.42$; moderate), and $4 \mathrm{~d}(r=-.28$; small) $(P<.05)$. Changes in HRex $(r=.28$; small; $P=.02)$ were correlated with changes in 4-d total high-speed-running-distance accumulation only. Correlations between variability in muscle soreness, sleep quality, CMJ, HRR\%, and HRV and total high-speed-running distance were negligible and not statistically significant for all accumulation training loads. Conclusions: Perceived ratings of fatigue and HRex were sensitive to fluctuations in acute total high-speed-running-distance accumulation, although sensitivity was not systematically influenced by the number of previous days over which the training load was accumulated. The present findings indicate that the sensitivity of morning-measured fatigue variables to changes in training load is generally not improved when compared with training loads beyond the previous day's training.
\end{abstract}

Keywords: training, performance, wellness, recovery

The locomotor demands of elite soccer have progressively increased in recent years. ${ }^{1,2}$ Since leading teams are also required to compete in a high number of matches over the course of season, ${ }^{3}$ implementation of effective recovery strategies are paramount to avoid the debilitating effects associated with overtraining and injury. ${ }^{4}$ Increasing attention in the literature has therefore focused on evaluating the effectiveness of a range of monitoring tools that may serve as valid indicators of fatigue status of athletes. ${ }^{5}$ For the purpose of this manuscript, fatigue will be defined as an inability to complete a task that was once achievable within a recent time frame. ${ }^{6}$

Recent research has examined the sensitivity of potential measures of fatigue to daily fluctuations in training load in Australian Rules Football (AFL) ${ }^{7,8}$ In AFL players, perceived ratings of wellness, ${ }^{7,8}$ submaximal heart rate (HRex), ${ }^{7}$ and an index (LnSD1) of vagal-related heart-rate variability $(\mathrm{HRV})^{7}$ were sensitive to the fluctuations in daily training load during a preseason training period. Similarly, in elite soccer players competing in the English Premier League (EPL), ${ }^{9}$ both rating of perceived fatigue and vagal related HRV measure Ln rMSSD were most sensitive to the

Thorpe and Strudwick are with the Medicine and Science Dept, Manchester United, Manchester, UK. Buchheit is with the Performance Dept, Paris Saint-Germain Football Club, Saint-Germain-en-Laye, France. Atkinson is with the Health and Social Care Inst, Teesside University, Teesside, UK. Drust and Gregson are with the Research Inst for Sport and Exercise Sciences, Liverpool John Moores University, Liverpool, UK. Address author correspondence to Robin T. Thorpe at robin.thorpe@manutd.co.uk. previous days fluctuations in training load experienced during the in-season competition period. Furthermore, in the same population, only perceived ratings of wellness were sensitive to within-week fluctuations in match and training load during typical in-season competition weeks. ${ }^{10}$ Collectively, these findings demonstrate that these measures, particular perceived ratings of wellness, show promise as acute, simple, noninvasive assessments for tracking the fatigue status of elite team-sport athletes.

Physiological adaptation to training is the culmination of repeated daily applications of training load. ${ }^{11}$ The level of fatigue experienced by an athlete at any single point in time is therefore unlikely to purely reflect the load incurred from the previous day's activity, ${ }^{9}$ but rather the load accumulated from a number of training days. Indeed high-intensity exercise and eccentric type activity leads to increases in muscle soreness that may be present for up to 72 hours after the exercise stress. ${ }^{12,13}$ In line with such observations, Buchheit ${ }^{14}$ recently suggested that $\mathrm{HRV}$ indices, used as an indicator of the athletes training status, may be more sensitive to changes in training loads when averaged across 7 days compared with a single daily measurement. Similarly, reductions and increases in heart-rate recovery (HRR) have been seen in response to weekly increases in training load and performance in physically active subjects and elite cyclists, respectively. ${ }^{15,16}$

Recent observations in elite senior soccer players have demonstrated that potential fatigue measures, particularly perceived ratings of wellness, were sensitive to within-week fluctuations in match and training load during typical competition weeks. ${ }^{9}$ Changes in these measures across the training week may, therefore, to some extent 
reflect the periodized training load incurred over a number of the preceding days and not solely the previous days training. It is possible therefore, that the relationship between such potential markers of fatigue and training load may vary as a function of the number of accumulated training days. The response to a single training session may not have the same physiological effect or magnitude compared with multiple training sessions performed over a short period of time. Therefore, our aim was to determine whether the sensitivity of a range of potential fatigue measures would vary when compared with the training load accumulated over the previous 2, 3, or 4 days during a short in-season competitive phase in elite soccer players. These data would enable comparison with previous observations in the same population which examined the sensitivity of the same measures to the previous day's fluctuations in training load. ${ }^{8}$

\section{Methods}

\section{Subjects}

Data were collected from 10 senior outfield soccer players (19.1 $\pm 0.6 \mathrm{y}, 1.84 \pm 0.7 \mathrm{~m}, 75.4 \pm 7.6 \mathrm{~kg}$ ) competing in the EPL over a 17-day period (February) during the in-season competition phase.

\section{Design}

Players took part in normal team training throughout the 17-day period as prescribed by the coaching staff. This included 2 competitive reserve team home matches (days 1 and 10), three rest days (day 6,11 , and 16) and 12 training sessions. All players were fully familiarized with the fatigue assessments in the weeks before completion of the main experimental trials. Fatigue measures were assessed each morning before the players commencing normal training. Perceived ratings of wellness measurements were assessed every day during the 17-day period. Physiological measurements were assessed every day with the exception of match and rest days. Each day players arrived at the training-ground laboratory having refrained from caffeine intake at least 12 hours before each assessment point. All assessments were conducted at the same time of the day to avoid the circadian variation in body temperature. ${ }^{17}$ Players were not allowed to consume fluid at any time during the fatigue assessments. The study was approved by the Liverpool John Moores University ethics committee. All players provided written informed consent. Before inclusion into the study, players were examined by the club physician and were deemed to be free from illness and injury.

\section{Methodology}

Individual player daily training and match load was monitored throughout the 17-day assessment period. Each player was also monitored during each training session and match using a portable global positioning system (GPS) technology (GPSports SPI ProX $5 \mathrm{~Hz}$, Canberra, Australia). This type of system has previously been shown to provide valid and reliable estimates of instantaneous velocity during acceleration, deceleration, and constant velocity movements during linear, multidirectional, and soccer-specific activities. ${ }^{18,19}$ All devices were activated 15 minutes before the data collection to allow acquisition of satellite signals. ${ }^{20}$ The minimum acceptable number of available satellite signals was 8 (range 8-11). ${ }^{21}$ Players wore the same GPS device for each session to avoid interunit error. ${ }^{21}$ Based on GPS data, locomotive speed above the threshold of $14.8 \mathrm{~km} / \mathrm{h}$ was classified as high-speed running. Total high-speedrunning distance was employed in the current study as an index of training and match load due to its frequent inclusion in attempts to quantify the load incurred by elite players during training and match play. ${ }^{22}$ However, high-speed running will underestimate the true load incurred by the athlete since it does not account for the stress associated with the frequent accelerations and decelerations which occur during soccer. ${ }^{23}$ It should be noted, however, that initial analysis in the current study highlighted a large correlation $(r=.57)$ between total high-speed-running distance and session ratings of perceived exertion (sRPE), which has previously been used as a global indicator of internal load in soccer players. ${ }^{24}$

\section{Perceived Ratings of Wellness}

A psychometric questionnaire was used daily before any training or exercise to assess general indicators of player wellness. ${ }^{9,10}$ The questionnaire comprised 3 questions relating to perceived sleep quality (coefficient of variation [CV] 13\%), muscle soreness (CV $9 \%$ ), and fatigue (CV 12\%). ${ }^{9}$ Each question scored on a 7-point Likert scale with scores of 1 to 7 with 1 and 7 representing very, very poor (negative state of wellness) and very, very good (positive state of wellness), respectively.

\section{Countermovement Jump}

Countermovement-jump ${ }^{9}$ (coefficient of variation [CV] $\left.4 \%\right)^{9}$ (CMJ) performance was evaluated using a jump mat (Fusion Sport, Queensland, Australia). Participants performed 5 CMJ efforts in total, 2 practice and 3 assessment jumps, ensuring that their hands were affixed to their hips throughout the jump. The highest jump was used as the criterion measure of performance.

\section{Heart-Rate Indices}

Players completed an indoor submaximal 5-minute cycling (Keiser, CA, USA), 5-minute recovery test as part of the warm-up before commencing every training session. ${ }^{9}$ All players were assessed together at a fixed exercise intensity of $130 \mathrm{~W}(85 \mathrm{rpm})$. This intensity was selected to minimize anaerobic energy contribution ${ }^{25}$ and to permit a rapid return of heart rate to baseline for short-term HRV measurements. On completion of exercise the players remained seated in silence for 5 minutes. HRV expressed as the square root of the mean of the sum of squares of differences between adjacent normal R-R intervals $(\mathrm{rMSSD}, \mathrm{CV} 28 \%)^{9}$ and the natural logarithm of the rMSSD (Ln rMSSD, CV 10\% $)^{9}$ were calculated as previously described ${ }^{25}$ using Polar software (Polar Precision Performance SW 5.20, Polar Electro, Kempele, Finland). HRR expressed as the absolute (HRR, CV 14\% $)^{9}$ and relative (\%HRR, CV 10\%) $)^{9}$ change in HR between the final 30 seconds (average) of the 5-minute cycling test and 60 seconds after cessation of exercise were calculated as previously described. ${ }^{9,16,25}$

\section{Statistical Analysis}

Data were analyzed with general linear models, which allowed for the fact that data were collected within-subjects over time. ${ }^{26}$ Recently, step-wise regression approaches have been criticized for reliable variable selection in a model. ${ }^{27,28}$ Our added problem was the predicted high multicollinearity between the various independent variables in our study. Therefore, we used a combination of expert knowledge regarding which variables hold superior practical/clinical importance ${ }^{28}$ and a multicollinearity correlation coefficient of $>.5$ for initial variable selection. Total high-speed-running distance was selected to provide an indication of training and match load 
(independent variable) in the current study. We then quantified the relationships between the various predictors and outcomes using model I (unadjusted model) and model II (fully adjusted model from which partial correlation coefficients and associated $95 \%$ confidence intervals for each predictor could be derived). To calculate acute training load accumulation, the rolling mean 2-, 3-, and 4-day total high-speed-running distances were then related to the subsequent day's morning-measured fatigue variables. The following criteria were adopted to interpret the magnitude of the correlation $(r)$ between test measures: <.1 trivial, .1 to .3 small, .3 to .5 moderate, .5 to .7 large, .7 to .9 very large, and .9 to 1.0 almost perfect. ${ }^{29}$ The level of statistical significance was set at $P<.05$ for all tests.

\section{Results}

Partial correlations, least-squares regression slope $(B)$, and significance for the relationship between total high-speed-running distance (over 2-4 d) and morning-measured fatigue variables are shown in Tables 1 to 7. Absolute variability in training load and fatigue measures over the 17-day period can be viewed in a recent publication ${ }^{9}$

Table 1 Partial Correlations (95\% Cl), Least-Squares Regression Slope $(B)$, and Significance for the Relationship Between Morning-Measured Perceived Fatigue and Total High-Speed-Running Distance Over the Previous 2, 3, and 4 Days

\begin{tabular}{lcccl}
\hline & $\begin{array}{c}\text { Correlation } \\
\text { coefficient }(\mathbf{9 5 \%} \mathbf{C l})\end{array}$ & Magnitude & $\boldsymbol{B}$ & $\boldsymbol{P}$ \\
\hline $2 \mathrm{~d}$ & $-.31(-.51$ to -.78$)$ & Small & 149.167 & .01 \\
$3 \mathrm{~d}$ & $-.42(-.61$ to -.18$)$ & Moderate & 166.509 & .001 \\
$4 \mathrm{~d}$ & $-.28(-.52$ to -.01$)$ & Small & 108.53 & .03 \\
\hline
\end{tabular}

Table 2 Partial Correlations (95\% Cl), Least-Squares Regression Slope $(B)$, and Significance for the Relationship Between Morning-Measured Perceived Sleep Quality and Total High-Speed-Running Distance Over the Previous 2, 3, and 4 Days

\begin{tabular}{lcccc}
\hline & $\begin{array}{c}\text { Correlation } \\
\text { coefficient }(\mathbf{9 5 \%} \mathbf{C l})\end{array}$ & Magnitude & $\boldsymbol{B}$ & $\boldsymbol{P}$ \\
\hline $2 \mathrm{~d}$ & $-.03(-.27$ to .21$)$ & Trivial & -10.633 & .826 \\
$3 \mathrm{~d}$ & $-.1(-.35$ to .16$)$ & Trivial & -9.869 & .813 \\
$4 \mathrm{~d}$ & $.04(-.27$ to .28$)$ & Trivial & 15.774 & .745 \\
\hline
\end{tabular}

Table 3 Partial Correlations (95\% Cl), Least-Squares Regression Slope $(B)$, and Significance for the Relationship Between Morning-Measured Perceived Muscle Soreness and Total High-Speed-Running Distance Over the Previous 2, 3, and 4 Days

\begin{tabular}{lcccc}
\hline & $\begin{array}{c}\text { Correlation } \\
\text { coefficient }(\mathbf{9 5 \%} \mathbf{~ C l})\end{array}$ & Magnitude & $\boldsymbol{B}$ & $\boldsymbol{P}$ \\
\hline $2 \mathrm{~d}$ & $-.19(-.41$ to .05$)$ & Trivial/Small & -58.443 & .12 \\
$3 \mathrm{~d}$ & $-.16(-.40$ to .10$)$ & Trivial & -36.258 & .227 \\
$4 \mathrm{~d}$ & $-.13(-.40$ to .15$)$ & Trivial & -28.05 & .365 \\
\hline
\end{tabular}

All players competed in both matches during the 17-day period with a median of 79 minutes playing time per player (range 32-93 min). Variability in ratings of perceived fatigue was correlated to variability in total high-speed-running distance covered on the previous 2,3 , and 4 days $(P<.05$; Table 1$)$. Small to moderate correlations were observed for 2- $(r=-.31), 3-(r=-.42)$, and 4-day $(r=-.28)$ cumulative total high-speed-running distance. Correlations between

Table 4 Partial Correlations (95\% Cl), LeastSquares Regression Slope (B), and Significance for the Relationship Between Morning-Measured Countermovement-Jump Performance and Total HighSpeed-Running Distance) Over the Previous 2, 3, and 4 Days

\begin{tabular}{lcccl}
\hline & $\begin{array}{c}\text { Correlation } \\
\text { coefficient }(\mathbf{9 5 \%} \mathbf{C l})\end{array}$ & Magnitude & $\boldsymbol{B}$ & $\boldsymbol{P}$ \\
\hline $2 \mathrm{~d}$ & $.13(-.11$ to .36$)$ & Trivial & 24.944 & .29 \\
$3 \mathrm{~d}$ & $.21(-.05$ to .42$)$ & Small & 31.478 & .109 \\
$4 \mathrm{~d}$ & $.23(-.05$ to .48$)$ & Small & 34.02 & .102 \\
\hline
\end{tabular}

Table 5 Partial Correlations $(95 \% \mathrm{Cl})$, Least-Squares Regression Slope (B), and Significance for the Relationship Between Morning-Measured Submaximal Heart Rate and Total High-Speed-Running Distance Over the Previous 2, 3, and 4 Days

\begin{tabular}{ccccc}
\hline & $\begin{array}{c}\text { Correlation } \\
\text { coefficient }(95 \% \mathrm{Cl})\end{array}$ & Magnitude & $\boldsymbol{B}$ & $\boldsymbol{P}$ \\
\hline $2 \mathrm{~d}$ & $.18(-.06$ to .40$)$ & Trivial & 5.17 & .1 \\
$3 \mathrm{~d}$ & $.21(-.05$ to .44$)$ & Small & 4.863 & .07 \\
$4 \mathrm{~d}$ & $.28(.05-.52)$ & Small & 5.948 & .02 \\
\hline
\end{tabular}

Table 6 Partial Correlations (95\% Cl), Least-Squares Regression Slope (B), and Significance for the Relationship Between Morning-Measured Ln rMSSD and Total High-Speed-Running Distance Over the Previous 2, 3, and 4 Days

\begin{tabular}{ccccc}
\hline & $\begin{array}{c}\text { Correlation } \\
\text { coefficient }(95 \% \text { Cl) }\end{array}$ & Magnitude & $\boldsymbol{B}$ & $\boldsymbol{P}$ \\
\hline $2 \mathrm{~d}$ & $<-.01(-.25$ to .29$)$ & Trivial & -1.31 & .99 \\
$3 \mathrm{~d}$ & $<.01(-.27$ to .25$)$ & Trivial & 9.426 & .907 \\
$4 \mathrm{~d}$ & $-.15(-.41$ to .13$)$ & Trivial & -95.337 & .279 \\
\hline
\end{tabular}

Table 7 Partial Correlations (95\% Cl), Least-Squares Regression Slope (B), and Significance for the Relationship Between Morning-Measured Heart-Rate Recovery and Total High-Speed-Running Distance Over the Previous 2, 3, and 4 Days

\begin{tabular}{ccccc}
\hline & $\begin{array}{c}\text { Correlation } \\
\text { coefficient }(\mathbf{9 5 \%} \mathbf{C l})\end{array}$ & Magnitude & $\boldsymbol{B}$ & $\boldsymbol{P}$ \\
\hline $2 \mathrm{~d}$ & $<.1(-.14$ to .33$)$ & Trivial & 0.178 & .970 \\
$3 \mathrm{~d}$ & $<.1(-.16$ to .35$)$ & Trivial & 1.138 & .762 \\
$4 \mathrm{~d}$ & $-.03(-.23$ to .32$)$ & Trivial & -1.584 & .684 \\
\hline
\end{tabular}


variability in perceived sleep quality and muscle soreness and total high-speed-running distance across all days were trivial to small and not statistically significant (Table 2 and 3).

Correlations between variability in CMJ and total high-speedrunning distance across all days were trivial to small and not statistically significant (Table 4).

Correlations between variability in HRex and total high-speedrunning distance across all days were trivial to small and only statistically significant with 4-day cumulative total high-speed-running distance $(r=.28 ; P=.02$; Table 5$)$.

Correlations between variability in HRR (\%) and Ln rMSSD and total high-speed-running distance for all days were trivial and not statistically significant (Table 6 and 7).

\section{Discussion}

The aim of the current study was to determine whether the sensitivity of a range of morning-measured fatigue variables to changes in training load was influenced by the number of previous days over which the training load was accumulated in elite soccer players. When compared with previous data published on the current population, the present findings indicate that the sensitivity of morning-measured fatigue measures to changes in training load is generally not improved when compared with training loads beyond the previous day's training. ${ }^{9}$

The use of simple perceived ratings of wellness is an efficient and practical approach to determining the fatigue status of elite team sport athletes. ${ }^{8-10,30}$ Previous observations on elite soccer players showed a moderate-to-strong significant correlation between the players perceived rating of fatigue and the previous days total high-speed-running distance $(r=-.51 ; P<.001) .{ }^{9}$ Furthermore, the slope of the regression model indicated that every $\sim 400-\mathrm{m}$ increase in total high-speed-running distance led to a 1-unit decrease (eg, a player may change from very poor level of fatigue to very, very poor level of fatigue after an additional $\sim 400$-m total high-speedrunning distance) in fatigue with $37 \%$ of the variance in training load explained by all the statistically significant predictors. ${ }^{9}$ In contrast, the current findings demonstrate that the sensitivity of morning-measured perceived fatigue, to changes in training load is reduced from significantly moderate to significantly small $(r=-.42$ to -.28 ) when compared with the training load observed beyond the previous days training ${ }^{9}$ Indeed, the variance in training load explained by all the statistically significant predictors decreased to $15 \%$ when training load was accumulated over a number (2-4) of days, highlighting the importance of immediately preceding load in elite soccer players. This apparent importance of the previous days training load on morning-measured fatigue may to some extent be explained by the nature of training cycles undertaken by elite soccer players. During the in-season competition period, players rotate around weekly cycles comprising one to two matches (very high load) interspersed with training sessions (moderate to high load) and recovery sessions. ${ }^{22,31,32}$ This cycle of daily loading peaks and-troughs within a short time frame may, therefore, only lead to changes in fatigue status that are largely representative of the previous days training. The influence of accumulated training load on morning measured perceived fatigue may be more relevant to endurance based sports where load is distributed and sustained over extended training blocks.

Small significant correlations have been reported between daily perceived ratings of sleep quality $(r=.2)$ and muscle soreness $(r=$ .3 ) and the previous days training load during preseason training in elite AFL players. ${ }^{7}$ In contrast, in EPL players the relationship between daily training load and perceived ratings of sleep quality and muscle soreness were trivial and nonsignificant. ${ }^{9}$ Furthermore, in the current study, we demonstrate that the magnitude of these relationships are not influenced by the number of days over which training load was accumulated. Muscle soreness has been found to be significantly elevated between 24 and 72 hours after a soccer match. ${ }^{12,13,33}$ Moreover, sleep quality has been seen to decrease around periods of competition. ${ }^{34}$ In the current study, only two match days were included in the sample of 17 days, consequently, the limited match exposure and training intensity may not have been sufficient to influence muscle soreness and sleep quality. In a previous study from the same population of players, match demands accounted for $\sim 40 \%$ of total weekly load, moreover, perceived ratings of wellness were found to be lowest on the day postmatch, ${ }^{10}$ further showing the debilitating effects of a match on fatigue status. Indeed, the average daily training load in the current study (RPE-TL 361 ) is considerably lower than that reported during an AFL preseason training camp (RPE-TL 746) where daily readings of muscle soreness and sleep quality were associated with changes in load. ${ }^{7}$ Future work involving a greater frequency of matches is therefore warranted to fully examine the influence of changes in loading on morning-measured perceived ratings of muscle soreness and sleep.

Previously, in elite soccer players, a small, positive daily correlation was observed $(r=.23)$ between $\mathrm{CMJ}$ height and total high-speed-running distance suggesting improved performance with increased total high-speed-running distance. ${ }^{9}$ It has been reported that the assessment of neuromuscular function via the use of jump protocols may be impaired up to 72 hours postmatch. ${ }^{35,36}$ However, in the current study, a nonsignificant trivial to small relationship was found between changes in CMJ height and total high-speedrunning distance accumulation over 2 to 4 days. Collectively, the findings from the current study and those from earlier investigations, ${ }^{9,37}$ demonstrate that CMJ height is generally insensitive to acute changes in workload in elite soccer players. CMJ height alone may be too crude of a measure to detect changes in training load, however, alternative CMJ derived neuromuscular parameters may hold sensitivity to alterations in load irrespective of the limited change in CMJ height. For example, neuromuscular parameters (eccentric, concentric, and total duration, time to peak force/power, flight-time:contraction-time ratio) derived from CMJ have been found suitable for detection of neuromuscular fatigue. ${ }^{38}$ Reductions in 18 different neuromuscular variables were found after a highintensity fatiguing protocol in college-level team-sport athletes. ${ }^{38}$ Furthermore, reductions in the flight-time:contraction-time ratio have been found across a season in AFL players indicating sensitivity to increases in load over time. ${ }^{39}$ Future research is required to investigate whether alternative measures derived from CMJ are sensitive to changes in training load in elite soccer players.

In recent years HR indices (HRV, HRR, and HRex) have been used as a popular method to measure variations in the autonomic nervous system (ANS) in an attempt to understand athlete adaptation/fatigue status. ${ }^{14}$ The use of vagal-related time-domain indices such as Ln rMSSD have been found to have greater reliability and are ideal for assessments over short periods when compared with spectral indices of HRV. ${ }^{40,41}$ A small significant correlation $(r=-.2$; $P=.04$ ) was found between the daily fluctuations in Ln rMSSD and total high-speed-running distance in elite soccer players from an earlier study. ${ }^{9}$ In this study, the slope of the regression model indicated that every $~ 300-\mathrm{m}$ increase in total high-speed-running distance led to a decrease of 1 unit in HRV, that is, more sympathetic dominance the greater the training load., ${ }^{7,42}$ In the current study, 
nonsignificant, trivial correlations were observed between fluctuations in 2-, 3-, and 4-day total high-speed-running distance and changes in morning-measured HRV, implying no additional effect on HRV beyond the previous days of training load. The limited relationships may reflect the low loads incurred by players observed in the current study. Buchheit et $\mathrm{al}^{7}$ found significant daily correlations $(r=.40)$ with a comparable vagal related parameter HRV (Ln SD1) during a preseason camp in AFL players. A possible reason for the small to moderate correlation found may be due to the enhanced training load performed by AFL players. ${ }^{7}$ Another potential reason for the lack of sensitivity observed for HRV in the current study may be due to the inherent variation of this measure. Indeed, based on data derived from endurance sports it is suggested that the use of a single data point could be misleading for practitioners due to the high day-to-day variation in these indices. ${ }^{43}$ When data were averaged over a week or using 7-day rolling averages, sensitivity to training load and performance has been improved compared with a single assessment point. A similar observation in young handball players has also been reported when single monthly assessments were found to have less than $20 \%$ sensitivity to training status. ${ }^{44}$ Future work is required to observe whether more frequent measures of HRV improve sensitivity to training load. Furthermore, future research is needed to establish how HRV responds to more extended and sustained periods of training and match load in elite soccer players.

In the current study, small significant increases in HRex were associated with increases in 4 days accumulated total high-speedrunning distance. In contrast, Buchheit et $\mathrm{al}^{7}$ found a large negative correlation between daily training load and HRex suggesting a reduction in heart rate after increases in training load. However, this data were collected during a short preseason AFL training camp in the heat where environmental and/or training induced changes in plasma volume are more likely responsible than alterations stemmed solely from the previous days training load. ${ }^{7}$ Reductions in heart rate have also been observed in athletes involved in extremely high training loads. ${ }^{45}$ Indeed, HRex during intensified training intensities showed significant reductions in overreached triathletes. Le Meur et $\mathrm{al}^{46,47}$ suggested the cause of this reduction in heart rate to be a hyperactivation of the parasympathetic nervous system via central, cardiac, and/or periphery mechanisms. In contrast to Le Meur et $\mathrm{al},{ }^{46,47}$ the results of the current study suggest, although, speculative, an acute stimulation of the sympathetic nervous system thus increasing HRex after a short continued period of training. Indeed, in both recreational marathon runners and world-class rowers, a significant increase in sympathetic dominance following a training block in the lead up to competition has been observed. ${ }^{48,49}$

Sensitivity between HRR\% and 2- to 4-day THIR accumulation was trivial and nonsignificant in the current study. Previous data also failed to find a relationship between daily HRR\% and total high-speed-running distance over a 17-day competitive period. ${ }^{9}$ In contrast, previous studies have observed responses between both acute and chronic training load and HRR. Borresen and Lambert ${ }^{15}$ found that HRR decreased with an increase in training load and subsequently a tendency for a faster HRR with a decrease in training load. The authors speculate, however, that the reduced HRR with an increase in training load may be explained by the severe increase in training load (TRIMP increased by 55\%), potentially inducing overreaching, and hence a parasympathetic predominance as previously discussed. ${ }^{45}$ The use of HRex and HRR in healthy athletes to predict changes in performance or fatigue should be treated with caution and interpreted together with other measures of fatigue, such as perceived ratings of wellness. ${ }^{14,50}$ As a consequence, if HRderived assessments of fatigue/adaptation are to be effective in team sports, a higher volume of assessments may be required as previously discussed. However, undertaking such measures may prove difficult with the large volume of athletes engaged in team sports. ${ }^{14}$

\section{Practical Applications}

Perceived ratings of fatigue show particular promise as simple, noninvasive assessments of fatigue status in elite soccer players during an in-season competitive phase. The present findings also indicate that the sensitivity of morning-measured fatigue variables to changes in training load is generally not improved when compared with training loads beyond the previous days training, therefore, it is likely to be most effective when taken on a daily basis. Future research is needed to determine the acute and longitudinal usefulness of HRex, HRR, and vagal related HRV as a monitoring tool in team sports.

\section{Conclusion}

The sensitivity of morning-measured fatigue variables to changes in training load is not improved when compared with training loads beyond the previous days training. Perceived ratings of fatigue shows the most promise as a simple, noninvasive assessment of fatigue status in elite soccer players in detection of acute load fluctuations during an in-season competitive phase compared with the other markers of fatigue measured.

\section{References}

1. Barnes C, Archer DT, Hogg B, Bush M, Bradley PS. The evolution of physical and technical performance parameters in the English Premier League. Int J Sports Med. 2014;35(13):1095-1100 doi:10.1055/s-0034-1375695. PubMed

2. Bradley PS, Archer DT, Hogg B, et al. Tier-specific evolution of match performance characteristics in the English Premier League: it's getting tougher at the top. J Sports Sci. 2016;34(10):980-987. doi:10.1080/0 2640414.2015.1082614 PubMed

3. Carling C, Gregson W, McCall A, Moreira A, Wong DP, Bradley PS. Match running performance during fixture congestion in elite soccer: research issues and future directions. Sports Med. 2015;45(5):605613. PubMed doi:10.1007/s40279-015-0313-z.

4. Nimmo MA, Ekblom B. Fatigue and illness in athletes. J Sports Sci. 2007;25(Suppl 1):S93-S102 doi:10.1080/02640410701607379. PubMed

5. Meeusen R, Duclos M, Foster C, et al. Prevention, diagnosis, and treatment of the overtraining syndrome: joint consensus statement of the European College of Sport Science and the American College of Sports Medicine. Med Sci Sports Exerc. 2013;45(1):186-205 doi:10.1249/MSS.0b013e318279a10a. PubMed

6. Halson SL. Monitoring training load to understand fatigue in athletes. Sports Med. 2014;44(Suppl 2):S139-S147 doi:10.1007/s40279-0140253-z. PubMed

7. Buchheit M, Racinais S, Bilsborough JC, et al. Monitoring fitness, fatigue and running performance during a pre-season training camp in elite football players. J Sci Med Sport. 2013;16(6):550-555 doi:10.1016/j.jsams.2012.12.003. PubMed

8. Gastin PB, Meyer D, Robinson D. Perceptions of wellness to monitor adaptive responses to training and competition in elite Australian football. J Strength Cond Res. 2013;27(9):2518-2526 doi:10.1519/ JSC.0b013e31827fd600. PubMed 
9. Thorpe RT, Strudwick AJ, Buchheit M, Atkinson G, Drust B, Gregson W. Monitoring fatigue during the in-season competitive phase in elite soccer players. Int J Sports Physiol Perform. 2015;10(8):958-964. doi:10.1123/ijspp.2015-0004 PubMed

10. Thorpe RT, Strudwick AJ, Buchheit M, Atkinson G, Drust B, Gregson W. The tracking of morning fatigue status across in-season training weeks in elite soccer players. Int J Sports Physiol Perform. 2016;11(7):947-952. doi:10.1123/ijspp.2015-0490. PubMed

11. Pyne DB, Mujika I, Reilly T. Peaking for optimal performance: research limitations and future directions. J Sports Sci. 2009;27(3):195-202 doi:10.1080/02640410802509136. PubMed

12. Ispirlidis I, Fatouros IG, Jamurtas AZ, et al. Time-course of changes in inflammatory and performance responses following a soccer game. Clin J Sport Med. 2008;18(5):423-431 doi:10.1097/ JSM.0b013e3181818e0b. PubMed

13. Fatouros IG, Chatzinikolaou A, Douroudos II, et al. Time-course of changes in oxidative stress and antioxidant status responses following a soccer game. J Strength Cond Res. 2010;24(12):3278-3286 doi:10.1519/JSC.0b013e3181b60444. PubMed

14. Buchheit M. Monitoring training status with HR measures: do all roads lead to Rome? Front Physiol. 2014;5:73. doi:10.3389/ fphys.2014.00073 PubMed

15. Borresen J, Lambert MI. Changes in heart rate recovery in response to acute changes in training load. Eur J Appl Physiol. 2007;101(4):503511 doi:10.1007/s00421-007-0516-6. PubMed

16. Lamberts RP, Swart J, Capostagno B, Noakes TD, Lambert MI. Heart rate recovery as a guide to monitor fatigue and predict changes in performance parameters. Scand J Med Sci Sports. 2010;20(3):449-457 doi:10.1111/j.1600-0838.2009.00977.x. PubMed

17. Reilly T, Brooks GA. Exercise and the circadian variation in body temperature measures. Int J Sports Med. 1986;7(6):358-362 doi:10.1055/s-2008-1025792. PubMed

18. Coutts AJ, Duffield R. Validity and reliability of GPS devices for measuring movement demands of team sports. J Sci Med Sport. 2010;13(1):133-135 doi:10.1016/j.jsams.2008.09.015. PubMed

19. Varley MC, Fairweather IH, Aughey RJ. Validity and reliability of GPS for measuring instantaneous velocity during acceleration, deceleration, and constant motion. J Sports Sci. 2012;30(2):121-127 doi:10.1080 /02640414.2011.627941. PubMed

20. Waldron M, Worsfold P, Twist C, Lamb K. Concurrent validity and test-retest reliability of a global positioning system (GPS) and timing gates to assess sprint performance variables. J Sports Sci. 2011;29(15):1613-1619 doi:10.1080/02640414.2011.608703. PubMed

21. Jennings D, Cormack S, Coutts AJ, Boyd LJ, Aughey RJ. Variability of GPS units for measuring distance in team sport movements. Int $J$ Sports Physiol Perform. 2010;5(4):565-569. PubMed doi:10.1123/ ijspp.5.4.565

22. Malone JJ, Di Michele R, Morgans R, Burgess D, Morton JP, Drust B. Seasonal training-load quantification in elite english premier league soccer players. Int J Sports Physiol Perform. 2015;10(4):489-497. doi:10.1123/ijspp.2014-0352 PubMed

23. Gaudino P, Iaia FM, Alberti G, Strudwick AJ, Atkinson G, Gregson W. Monitoring training in elite soccer players: systematic bias between running speed and metabolic power data. Int J Sports Med. 2013;34(11):963-968 doi:10.1055/s-0033-1337943. PubMed

24. Impellizzeri FM, Rampinini E, Coutts AJ, Sassi A, Marcora SM. Use of RPE-based training load in soccer. Med Sci Sports Exerc. 2004;36(6):1042-1047. PubMed doi:10.1249/01. MSS.0000128199.23901.2F

25. Buchheit M, Millet GP, Parisy A, Pourchez S, Laursen PB, Ahmaidi S. Supramaximal training and postexercise parasympathetic reactivation in adolescents. Med Sci Sports Exerc. 2008;40:362-371 doi:10.1249/ mss.0b013e31815aa2ee. PubMed

26. Bland JM, Altman DG. Statistical methods for assessing agreement between two methods of clinical measurement. Lancet. 1986;1(8476):307-310. PubMed doi:10.1016/S0140-6736(86)90837-8

27. Whittingham MJ, Stephens PA, Bradbury RB, Freckleton RP. Why do we still use stepwise modelling in ecology and behaviour? J Anim Ecol. 2006;75(5):1182-1189 doi:10.1111/j.1365-2656.2006.01141.x. PubMed

28. Flom P, Cassell D. Stopping stepwise: why stepwise and similar selection methods are bad, and what you should use. In: NESUG Stats and Data Analysis 2007. Available at: http://www.lexjansen.com/ pnwsug/2008/DavidCassell-StoppingStepwise.pdf.

29. Hopkins WG. Measures of reliability in sports medicine and science. Sports Med. 2000;30(1):1-15. PubMed doi:10.2165/ 00007256-200030010-00001

30. Hooper SL, Mackinnon LT, Howard A, Gordon RD, Bachmann AW. Markers for monitoring overtraining and recovery. Med Sci Sports Exerc. 1995;27(1):106-112. PubMed doi:10.1249/ 00005768-199501000-00019

31. Anderson L, Orme P, Di Michele R, et al. Quantification of training load during one-, two- and three-game week schedules in professional soccer players from the English Premier League: implications for carbohydrate periodisation. J Sports Sci. 2016;34(13):1250-1259 doi:10.1080/0264 0414.2015.1106574. PubMed

32. Anderson L, Orme P, Di Michele R, et al. Quantification of seasonallong physical load in soccer players with different starting status from the English Premier League: implications for maintaining squad physical fitness. Int J Sports Physiol Perform. 2016;11(8):1038-1046. doi:10.1123/ijspp.2015-0672 PubMed

33. Magalhães J, Rebelo A, Oliveira E, Silva JR, Marques F, Ascensão A. Impact of Loughborough Intermittent Shuttle Test versus soccer match on physiological, biochemical and neuromuscular parameters. Eur J Appl Physiol. 2010;108(1):39-48 doi:10.1007/s00421-009-1161-z. PubMed

34. Lastella M, Roach GD, Halson SL, Martin DT, West NP, Sargent C. Sleep/wake behaviour of endurance cyclists before and during competition. J Sports Sci. 2015;33(3):293-299 doi:10.1080/02640414.2014.9 42690. PubMed

35. Silva JR, Rebelo A, Marques F, et al. Biochemical impact of soccer: an analysis of hormonal, muscle damage, and redox markers during the season. Appl Physiol Nutr Metab. 2014;39(4):432-438 doi:10.1139/ apnm-2013-0180. PubMed

36. Cormack SJ, Newton RU, McGuigan MR. Neuromuscular and endocrine responses of elite players to an Australian Rules football match. Int J Sports Physiol Perform. 2008;3(3):359-374. PubMed doi:10.1123/ ijspp.3.3.359

37. Malone JJ, Murtagh C, Morgans R, Burgess D, Morton JP, Drust B. Countermovement jump performance is not affected during an in-season training microcycle in elite youth soccer players. J Strength Cond Res. 2015;29(3):752-757. 10.1519/JSC.0000000000000701 PubMed

38. Gathercole R, Sporer B, Stellingwerff T, Sleivert G. Alternative countermovement-jump analysis to quantify acute neuromuscular fatigue. Int J Sports Physiol Perform. 2015;10(1):84-92 doi:10.1123/ ijspp.2013-0413. PubMed

39. Cormack SJ, Newton RU, McGuigan MR, Cormie P. Neuromuscular and endocrine responses of elite players during an Australian rules football season. Int J Sports Physiol Perform. 2008;3(4):439-453. PubMed doi:10.1123/ijspp.3.4.439

40. Al Haddad H, Laursen PB, Chollet D, Ahmaidi S, Buchheit M. Reliability of resting and postexercise heart rate measures. Int J Sports Med. 2011;32(8):598-605. PubMed doi:10.1055/s-0031-1275356 
41. Esco MR, Flatt AA. Ultra-short-term heart rate variability indexes at rest and post-exercise in athletes: evaluating the agreement with accepted recommendations. J Sports Sci Med. 2014;13(3):535-541. PubMed

42. Plews DJ, Laursen PB, Kilding AE, Buchheit M. Heart rate variability in elite triathletes, is variation in variability the key to effective training: a case comparison. Eur J Appl Physiol. 2012;112(11):3729-3741 doi:10.1007/s00421-012-2354-4. PubMed

43. Plews DJ, Laursen PB, Stanley J, Kilding AE, Buchheit M. Training adaptation and heart rate variability in elite endurance athletes: opening the door to effective monitoring. Sports Med.2013;43(9):773-781 doi:10.1007/s40279-013-0071-8. PubMed

44. Buchheit M. Sensitivity of monthly heart rate and psychometric measures for monitoring physical performance in highly trained young handball players. Int J Sports Med. 2015;36(5):351-356. doi:10.1055/s-0034-1385882 PubMed

45. Le Meur Y, Louis J, Aubry A, et al. Maximal exercise limitation in functionally overreached triathletes: role of cardiac adrenergic stimulation. J Appl Physiol. 2014;117(3):214-222 doi:10.1152/japplphysiol.00191.2014. PubMed
46. Le Meur Y, Pichon A, Schaal K, et al. Evidence of parasympathetic hyperactivity in functionally overreached athletes. Med Sci Sports Exerc. 2013;45(11):2061-2071. doi:10.1249/MSS.0b013e3182980125

47. Le Meur Y, Hausswirth C, Natta F, Couturier A, Bignet F, Vidal PP. A multidisciplinary approach to overreaching detection in endurance trained athletes. J Appl Physiol. 2013;114(3):411-420 doi:10.1152/ japplphysiol.01254.2012. PubMed

48. Manzi V, Castagna C, Padua E, et al. Dose-response relationship of autonomic nervous system responses to individualized training impulse in marathon runners. Am J Physiol Heart Circ Physiol. 2009;296(6):H1733-H1740 doi:10.1152/ajpheart.00054.2009. PubMed

49. Iellamo F, Pigozzi F, Spataro A, Lucini D, Pagani M. T-wave and heart rate variability changes to assess training in world-class athletes. Med Sci Sports Exerc. 2004;36(8):1342-1346. PubMed doi:10.1249/01. MSS.0000135796.75091.8A

50. Buchheit M, Simpson MB, Al Haddad H, Bourdon PC, MendezVillanueva A. Monitoring changes in physical performance with heart rate measures in young soccer players. Eur J Appl Physiol. 2012;112(2):711-723 doi:10.1007/s00421-011-2014-0. PubMed 